\title{
Correction: Global Changes and Factors of Increase in Caloric/Salty Food Intake, Screen Use, and Substance Use During the Early COVID-19 Containment Phase in the General Population in France: Survey Study
}

Benjamin Rolland $^{1,2}, \mathrm{MD}, \mathrm{PhD}$; Frédéric Haesebaert ${ }^{3}, \mathrm{MD}, \mathrm{PhD}$; Elodie Zante ${ }^{3}, \mathrm{MD}$; Amine Benyamina ${ }^{4,5}, \mathrm{MD}, \mathrm{PhD}$; Julie Haesebaert ${ }^{6}, \mathrm{MD}, \mathrm{PhD}$; Nicolas Franck ${ }^{5}, \mathrm{MD}, \mathrm{PhD}$

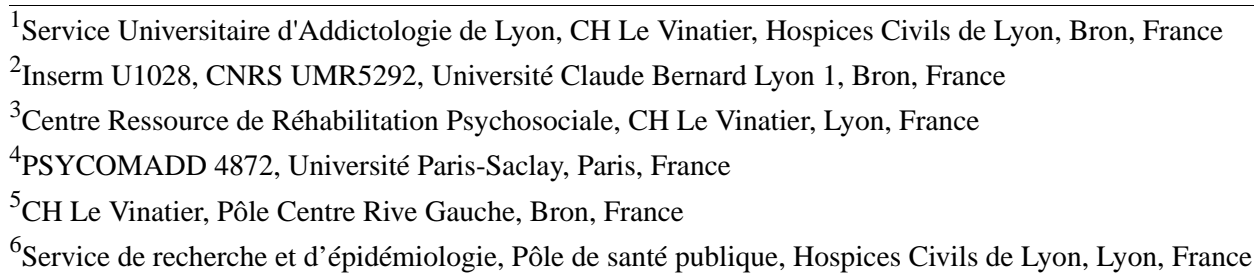

\section{Corresponding Author:}

Benjamin Rolland, MD, PhD

Service Universitaire d'Addictologie de Lyon

$\mathrm{CH}$ Le Vinatier

Hospices Civils de Lyon

Bâtiment 502

Bron, 69500

France

Phone: 330437915075

Email: benjrolland@gmail.com

\section{Related Article:}

Correction of: http://mhealth.jmir.org/2020/3/e19630/

(JMIR Public Health Surveill 2021;7(7):e31906) doi: 10.2196/31906

In "Global Changes and Factors of Increase in Caloric/Salty Food Intake, Screen Use, and Substance Use During the Early COVID-19 Containment Phase in the General Population in France: Survey Study" (JMIR Public Health Surveill 2020;6(3):e19630), one error was noted.

In the originally published article, one paragraph in the Results section incorrectly referred to "alcohol use" instead of "cannabis use." The full paragraph was published as follows:

Finally, regarding cannabis use, 620/11,391 (5.44\%) participants reported using cannabis. Among the, 263/620 (39.49\%) reported that they had not changed their average daily use of alcohol, whereas 162 $(24.32 \%)$ declared having moderately increased their alcohol use, 46 (6.91\%) increased their alcohol use in a difficult-to-control manner, 150 (22.52\%) reduced or stopped their alcohol use without craving/withdrawal, and $45(6.76 \%)$ reduced their alcohol use with craving/withdrawal.
This paragraph has been corrected as follows:

Finally, regarding cannabis use, 620/11,391 (5.44\%) participants reported using cannabis. Among the, 263/620 (39.49\%) reported that they had not changed their average daily use of cannabis, whereas 162 (24.32\%) declared having moderately increased their cannabis use, 46 (6.91\%) increased their cannabis use in a difficult-to-control manner, 150 (22.52\%) reduced or stopped their cannabis use without craving/withdrawal, and $45(6.76 \%)$ reduced their cannabis use with craving/withdrawal.

The correction will appear in the online version of the paper on the JMIR Publications website on July 20, 2021, together with the publication of this correction notice. Because this was made after submission to PubMed, PubMed Central, and other full-text repositories, the corrected article has also been resubmitted to those repositories. 
This is a non-peer-reviewed article. Submitted 08.07.21; accepted 08.07.21; published 20.07.21.

Please cite as:

Rolland B, Haesebaert F, Zante E, Benyamina A, Haesebaert J, Franck $N$

Correction: Global Changes and Factors of Increase in Caloric/Salty Food Intake, Screen Use, and Substance Use During the Early

COVID-19 Containment Phase in the General Population in France: Survey Study

JMIR Public Health Surveill 2021;7(7):e31906

URL: https://publichealth.jmir.org/2021/7/e31906

doi: $10.2196 / 31906$

PMID: $\underline{34283724}$

(CBenjamin Rolland, Frédéric Haesebaert, Elodie Zante, Amine Benyamina, Julie Haesebaert, Nicolas Franck. Originally published in JMIR Public Health and Surveillance (https://publichealth.jmir.org), 20.07.2021. This is an open-access article distributed under the terms of the Creative Commons Attribution License (https://creativecommons.org/licenses/by/4.0/), which permits unrestricted use, distribution, and reproduction in any medium, provided the original work, first published in JMIR Public Health and Surveillance, is properly cited. The complete bibliographic information, a link to the original publication on https://publichealth.jmir.org, as well as this copyright and license information must be included. 